Pacific

Journal of

Mathematics

APPLYING FUNCTIONAL IDENTITIES TO SOME LINEAR PRESERVER PROBLEMS

K.I. Beidar, M. Brešar, M.A. Chebotar, and Y. Fong 


\title{
APPLYING FUNCTIONAL IDENTITIES TO SOME LINEAR PRESERVER PROBLEMS
}

\author{
K.I. Beidar, M. Brešar, M.A. Chebotar, and Y. Fong \\ The theory of functional identities is used to obtain alge- \\ braic generalizations of some operator-theoretic results con- \\ cerning commutativity and normal preserving linear maps be- \\ tween algebras with involution.
}

\section{Introduction.}

Over the last decades there has been a considerable interest in linear algebra and operator theory in the so-called linear preserver problems (see survey articles $[\mathbf{1}, \mathbf{1 3}, \mathbf{1 9}, \mathbf{2 0}])$. By a linear preserver we mean a linear map of algebras which, roughly speaking, preserve certain properties of some elements in an algebra. In the literature these algebras are usually algebras of matrices or algebras of bounded linear operators. The goal in the study of linear preservers is to find their form. It turns out that often the only solutions are just the most obvious ones, frequently (anti)isomorphisms or at least maps related to them.

It is our impression that some linear preserver problems could be solved in a more general setting using only ring-theoretic techniques. An example illustrating this general conjecture is a characterization of bijective linear maps of prime algebras that preserve commutativity, i.e., they map commuting pairs of elements into commuting pairs [10, Theorem 2] (see also $[2,4]$ for some generalizations). This characterization was known before only for some special prime algebras which are studied in linear algebra and operator theory (see [10] for references). Moreover, it has turned out that one does not really need to assume that the map, say $\theta$, preserves the commutativity of all elements, but only that $\theta(x)$ and $\theta\left(x^{2}\right)$ commute for every $x$. The fact that only this milder condition has to be asssumed has proved to be useful when this result was applied to another linear preserver problem, namely, the one concerning maps on the algebra of bounded linear operators on a Hilbert space that preserve normal operators [12].

The proof of [10, Theorem 2] was based on a characterization of commuting traces of biadditive maps [10, Theorem 1], which was one of the first results in the area which is now called the theory of functional identities in rings. Over the last few years this theory has been systematically developed. It is our goal in this paper to show that some of its most recent results 
$[\mathbf{5}, \mathbf{6}, \mathbf{7}]$ can be used to obtain some further improvements in the study of linear maps preserving commutativity or normal elements.

First we introduce some terminology and fix the notation. A prime algebra $A$ over a field $F$ is said to be centrally closed over $F$ if both the center and the extended centroid of $A$ are equal to $F$. We will consider centrally closed prime algebras $A, A^{\prime}$ over $F$ with involution $*$ (by an involution we mean an additive involution, that is, $*$ satisfies $(x+y)^{*}=x^{*}+y^{*}$, $(x y)^{*}=y^{*} x^{*}$ and $\left.\left(x^{*}\right)^{*}=x\right)$. We say that a linear map $\theta: A \rightarrow A^{\prime}$ is *-linear if $\theta\left(x^{*}\right)=\theta(x)^{*}$ for all $x \in A$. Let $S=\left\{x \in A \mid x^{*}=x\right\}$ be the set of all symmetric elements in $A$, and $K=\left\{x \in A \mid x^{*}=-x\right\}$ be the set of all skew elements in $A$. Similarly, by $S^{\prime}$ and $K^{\prime}$ we denote the sets of all symmetric and skew elements in $A^{\prime}$, respectively. Next we set $F_{s}=F \cap S$. We say that the involution $*$ is of the first kind if $F=F_{s}$ (equivalently, $*$ is $F$-linear); otherwise we say that $*$ is of the second kind. Given a subset $R$ of $A$, we write $\langle R\rangle$ for the subalgebra of $A$ generated by $R$.

The concepts of the extended centroid and centrally closed prime algebras are explained in detail in the book [8]. Nevertheless, as some readers may be primarily interested in what is the meaning of our results in linear algebra and operator theory, let us just mention that the algebras of square matrices over a division ring, bounded linear operators on a Banach space (and moreover, all its subalgebras containing the identity and all finite rank operators) and prime unital $C^{*}$-algebras (in particular, von Neumann factors) are all examples of prime algebras centrally closed over their centers. Also, if one wants to restrict the attention to the case when $A$ and $A^{\prime}$ are algebras consisting of linear operators on a Hilbert space $H$ and $x^{*}$ is the adjoint of the operator $x$, then $*$ is of the first kind when $H$ is a real space, and $*$ is of the second kind when $H$ is a complex space.

In Section 2 we gather together some results of the theory of functional identities that are needed later on.

In Section 3 we extend the treatment of commutativity-preserving maps of prime algebras [10] by considering maps from $S$ onto $S^{\prime}$. The result which we obtain is a ring-theoretic extension of the results on maps preserving commutativity of symmetric matrices (operators) $[\mathbf{1 5}, \mathbf{1 4}, \mathbf{2 4}]$. Actually, as in [10], we do not really assume that the map $\theta$ preserves the commutativity of all elements in $S$, but only that $\theta(s)$ and $\theta\left(s^{2}\right)$ commute for every $s \in S$.

In Section 4 we consider bijective linear maps of algebras with involution of the second kind which preserve normal elements. As already mentioned, the special case when the algebras under consideration are algebras of all bounded linear operators on a complex Hilbert space was treated in [12] (see also $[\mathbf{1 5}, \mathbf{1 6}])$. It has turned out that Fuglede's theorem [25, Corollary 1.18], upon which the proof in [12] depends, can be avoided when treating this problem, and so we will be able to prove a ring-theoretic generalization of the result of $[\mathbf{1 2}]$. 
The problem of describing normal-preserving maps is much more difficult when the involution is of the first kind. First of all, the involution is then a linear operator and so, for instance, a map of the form $x \mapsto \mu_{1} x+\mu_{2} x^{*}$, where $\mu_{1}, \mu_{2} \in F$, is linear and preserves normal elements. Thus, we cannot expect the same result as in Section 4. Moreover, consider the following example.

Let $A=F\langle x, y\rangle$, where $F$ is a field, be a free algebra in two indeterminates $x$ and $y$ (incidentally, $A$ is a centrally closed prime algebra [8, Theorem 2.4.4]), and equip $A$ with standard involution (given by $x^{*}=x, y^{*}=y$ and $\left.\lambda^{*}=\lambda, \lambda \in F\right)$. Let $U$ be a linear span of all monomials in which both $x$ and $y$ appear, and $V$ be a linear span of all monomials $x^{n}, y^{n}$ with $n \geq 1$ (in particular, $V \subset S$ ). Then $A=F \oplus U \oplus V$ and note that a nonzero element in $U$ never commutes with a nonzero element in $V$. Now let $T: V \rightarrow V$ be any bijective linear operator. Then the map $A \rightarrow A$ defined by $\lambda+u+v \mapsto \lambda+u+T(v)$ is $*$-linear, bijective and maps normal elements onto normal elements.

This example somehow indicates that it is almost impossible to obtain a definitive result for preservers of normal elements in the case the involution is of the first kind. Nevertheless, even in this example the map acts very simply on a rather large piece of $A$, namely on $F \oplus U$. In Section 5 we shall see that under some technical conditions (in particular, we have to assume that our map is $*$-linear) the action of normal-preservers can be described on $\langle K\rangle$, which can certainly be considered as a "large piece" of $A$. In particular, except in some very special case, it contains a nonzero ideal of $A$ [8, Lemma 9.1.4 and Corollary 9.1.8]. Therefore, in simple algebras satisfying our technical assumptions, normal-preserving maps can be completely determined.

\section{Preliminaries.}

The aim of this section is to give a brief and self-contained outline of some parts the theory of functional identities, namely, those parts that shall really be needed in the subsequent sections. For a more detailed account on this theory we refer the reader to $[\mathbf{1 1}]$.

Throughout, the denotations $F, F_{s}, A, K, S, A^{\prime}, K^{\prime}$ and $S^{\prime}$ shall have the meaning already explained in the introduction. Though not always needed, we assume for simplicity that $\operatorname{char}(F) \neq 2$. Given $x, y \in A$, we set

$$
[x, y]=x y-y x \quad \text { and } \quad x \circ y=x y+y x .
$$

Next, by $\operatorname{deg}(x)$ we shall mean the degree of $x$ over $F$ (if $x$ is algebraic over $F$ ) or $\infty$ (if $x$ is not algebraic over $F$ ). Next we set

$$
\operatorname{deg}(A)=\sup \{\operatorname{deg}(x) \mid x \in A\} .
$$


For instance, $\operatorname{deg}\left(M_{n}(F)\right)=n$ for any field $F$. Moreover, from the structure theory of rings with polynomial identities $[\mathbf{2 3}, \mathbf{2 6}]$ it can be deduced that $\operatorname{deg}(A)=n<\infty$ if and only if $A$ is a subring of $M_{n}(\bar{F})$ such that $\bar{F} A=$ $M_{n}(\bar{F})$, where $\bar{F}$ is the algebraic closure of $F$.

The goal in the study of functional identities is, roughly speaking, to describe the form of maps satisfying certain identities. The first functional identities that have been considered were those concerned with the so-called commuting maps, i.e., maps whose values commute with the variable. Let us now reword the basic result on commuting maps [9, Theorem 3.2] in the following somewhat unusual but useful form.

Theorem 2.1. Let $f, \theta: A \rightarrow A^{\prime}$ be linear maps such that $[f(x), \theta(x)]=0$ for all $x \in A$. If $\theta$ is bijective, then there is $\tau \in F$ and a linear map $\zeta: A \rightarrow F$ such that $f(x)=\tau \theta(x)+\zeta(x)$ for all $x \in A$.

Actually, [9] considers only the case when $A=A^{\prime}$ and $\theta$ is the identity map. However, the seemingly more general condition treated in Theorem 2.1 can be reduced to that one by replacing the map $f$ by the map $f \theta^{-1}$. We also remark that in $[\mathbf{9}]$ the result is stated for additive maps on rings and not linear maps on algebras, but the necessary modifications in the proof are obvious. The same remarks apply for the remaining results in this section. Moreover, in these three theorems such terms as linearity and vector space should be understood with respect to the field $F_{s}$ rather than $F$.

A map $q: A \rightarrow A^{\prime}$ is said to be a trace of a $k$-linear map if there is a map $B: A^{k} \rightarrow A^{\prime}$, linear in each argument and such that $q(x)=B(x, \ldots, x)$ for all $x \in A$ (by a trace of a 0 -linear map we shall mean a constant). In the case when $\operatorname{char}(F)=0$ or $\operatorname{char}(F)>k$, there is no loss of generality in assuming that this map $B$ is symmetric (namely, otherwise replace $B\left(x_{1}, \ldots, x_{k}\right.$ ) by $\left.\frac{1}{k !} \sum_{\pi \in S_{k}} B\left(x_{\pi(1)}, \ldots, x_{\pi(k)}\right)\right)$.

The next theorem follows from [7, Theorem 5.5] and [5, Lemma 2.2].

Theorem 2.2. Let $R$ be a vector subspace of $A$, and let $R^{\prime}$ be either $S^{\prime}$ or $K^{\prime}$. Suppose that a trace of an n-linear map $q: R \rightarrow A^{\prime}$ satisfies

$$
\sum_{i=0}^{m} \mu_{i} \theta(x)^{i} q(x) \theta(x)^{m-i}=0 \quad \text { for all } x \in R,
$$

where $\mu_{0}, \ldots, \mu_{m}$ belong to $F$ and not all of them are 0 , and $\theta: R \rightarrow R^{\prime}$ is a bijective linear map. Suppose that $\operatorname{char}(F)=0$ or $\operatorname{char}(F)>n$ and $\operatorname{deg}\left(A^{\prime}\right)>2(m+n)$. Then:

(i) $q(x)=\sum_{k=0}^{n} \lambda_{k}(x) \theta(x)^{n-k}, x \in R$, where each $\lambda_{k}: R \rightarrow F$ is a trace of a $k$-linear map;

(ii) if $\sum_{i=0}^{m} \mu_{i} \neq 0$, then $q=0$.

Keeping the notation of Theorem 2.2, assume that $q(x) \theta(x) \in F$ for all $x \in R$, where $q(x)=B(x, \ldots, x)$ and $B$ is an $n$-linear map. A standard 
approach, the so-called complete linearization, then shows that

$$
\sum_{\pi \in S_{n+1}} B\left(x_{\pi(1)}, \ldots, x_{\pi(n)}\right) \theta\left(x_{\pi(n+1)}\right) \in F .
$$

Applying [7, Lemma 4.3] together with [5, Lemma 2.2] we then obtain:

Theorem 2.3. Let $R$ be a vector subspace of $A$, and let $R^{\prime}$ be either $S^{\prime}$ or $K^{\prime}$. Suppose that $q: R \rightarrow A^{\prime}$ is a trace of an n-linear map $B: R^{n} \rightarrow A^{\prime}$ such that $q(x) \theta(x) \in F$ for all $x \in R($ or $\theta(x) q(x) \in F$ for all $x \in R)$, where $\theta: R \rightarrow R^{\prime}$ is a bijective linear map. If $\operatorname{deg}\left(A^{\prime}\right)>2 n+2$, then $\sum_{\pi \in S_{n}} B\left(x_{\pi(1)}, \ldots, x_{\pi(n)}\right)=0$. Thus, if $\operatorname{char}(F)=0$ or $\operatorname{char}(F)>n$, then $q=0$.

We conclude this section with a result which might appear somewhat strange. However, the conditions treated in this result really appear in the proof of Theorem 5.1.

Theorem 2.4. Let $f\left(x_{1}, \ldots, x_{m}\right)$ be a multilinear polynomial in noncommuting variables $x_{1}, \ldots, x_{m}$ such that $f\left(k_{1}, \ldots, k_{m}\right) \in K$ for all $k_{1}, \ldots, k_{m}$ $\in K$. Let $\phi$ be a linear map of $K$ onto $K^{\prime}$ such that

$$
\phi\left(f\left(k_{1}, \ldots, k_{m}\right)\right)=\lambda f\left(\phi\left(k_{1}\right), \ldots, \phi\left(k_{m}\right)\right) \quad \text { for all } k_{1}, \ldots, k_{m} \in K,
$$

where $\lambda$ is a nonzero element in $F$. Further, let a map $B: K \times K \rightarrow A^{\prime}$ be such that

$$
B(k, l)=-B(l, k)
$$

for all $k, l \in K$, and

$$
\begin{aligned}
& B\left(f\left(k_{1}, \ldots, k_{m}\right), l\right) \\
& =\sum_{i=1}^{m} \lambda f\left(\phi\left(k_{1}\right), \ldots, \phi\left(k_{i-1}\right), B\left(k_{i}, l\right), \phi\left(k_{i+1}\right), \ldots, \phi\left(k_{m}\right)\right)
\end{aligned}
$$

for all $k_{1}, \ldots, k_{m}, l \in K$. If $\operatorname{deg}\left(A^{\prime}\right)>4 m+1$, then there exists $\rho \in F$ such that

$$
B(k, l)-\rho[\phi(k), \phi(l)] \in F \quad \text { for all } k, l \in K .
$$

For $\lambda=1$, Theorem 2.4 can be deduced at once from the statements of [5, Theorems 2.4] and [6, Theorem 2.9]. Almost the same proof, however, still works in the case when $\lambda$ is any nonzero element in $F$.

We have seen that excluding algebras of "small" degree one can obtain definite results on functional identities. As a consequence, the proofs of our main results will work as long as the degree of the algebra will be big enough. 


\section{Commutativity-preservers on symmetric elements.}

Having Theorems 2.2 and 2.3 in hand, the following result can be easily obtained just by modifying the proof of [10, Theorem 2].

Theorem 3.1. Let $A$ be $A^{\prime}$ be centrally closed prime algebras over a field $F$ with involution. Let $\theta: S \rightarrow S^{\prime}$ be a bijective $F_{s}$-linear map such that $\theta(s)$ and $\theta\left(s^{2}\right)$ commute for every $s \in S$. Suppose that $\operatorname{deg}(A)>4, \operatorname{deg}\left(A^{\prime}\right)>8$ and $\operatorname{char}(F) \neq 2,3$. Then $\theta$ is of the form $\theta(s)=\alpha \phi(s)+\beta(s)$ where $\alpha \in F_{s}, \alpha \neq 0, \beta$ is a $F_{s}$-linear map from $S$ into $F_{s}$ and $\phi:\langle S\rangle \rightarrow\left\langle S^{\prime}\right\rangle$ is an $F_{s}$-algebra isomorphism.

Proof. We have $\theta(s) \theta\left(s^{2}\right)-\theta\left(s^{2}\right) \theta(s)=0$ for all $s \in S$. Clearly, the map $s \mapsto \theta\left(s^{2}\right)$ is a trace of a bilinear map and so Theorem 2.2 implies that

$$
\theta\left(s^{2}\right)=\lambda \theta(s)^{2}+\mu(s) \theta(s)+\nu(s)
$$

where $\lambda \in F, \mu: A \rightarrow F$ is a linear and $\nu: A \rightarrow F$ is a trace of a bilinear map (again, the term linearity refers to $F_{s}$ and not $F$ ). We claim that $\lambda \in F_{s}$ and $\mu(s), \nu(s) \in F_{s}$ for all $s \in S$. Indeed, since both $\theta(s)$ and $\theta\left(s^{2}\right)$ are symmetric for any $s \in S$, it follows that

$$
\lambda \theta(s)^{2}+\mu(s) \theta(s)+\nu(s)=\lambda^{*} \theta(s)^{2}+\mu(s)^{*} \theta(s)+\nu(s)^{*}
$$

and so

$$
\left\{\left(\lambda-\lambda^{*}\right) \theta(s)+\mu(s)-\mu(s)^{*}\right\} \theta(s) \in F \quad \text { for all } s \in S .
$$

Since $\operatorname{deg}\left(A^{\prime}\right)$ is, in particular, $>4$, Theorem 2.3 first gives

$$
\left(\lambda-\lambda^{*}\right) \theta(s)+\mu(s)-\mu(s)^{*}=0 \quad \text { for all } s \in S,
$$

which in turn implies, again by Theorem 2.3, that $\lambda=\lambda^{*}$ and $\mu(s)=\mu(s)^{*}$. But then also $\nu(s)=\nu(s)^{*}$ for any $s \in S$.

Next we claim that $\theta(1)$ is a central element, that is, it lies in $F_{s}$. Just as in $\left[\mathbf{1 0}\right.$, p. 535], substituting $s+1$ for $s$ in $\left[\theta\left(s^{2}\right), \theta(s)\right]=0$ we arrive at $\left[\theta\left(s^{2}+s\right), \theta(1)\right]=0$, and then repeating the same substitution we get that $[\theta(s), \theta(1)]=0$ for all $s \in S$. But then, since $\operatorname{deg}\left(A^{\prime}\right)>2$, applying Theorem 2.2 again (or just referring to the standard theory of rings with involution) we infer that $\theta(1)$ is central.

Suppose that $\lambda=0$. Then, since $\theta$ is $F_{s}$-linear, it follows that $\theta\left(s^{2}-\right.$ $\mu(s) s) \in F_{s}$, which in turn implies, again using the $F_{s}$-linearity of $\theta$ together with $\theta(1) \in F_{s}$, that $(s-\mu(s)) s \in F_{s}$ for all $s \in S$. Since $\operatorname{deg}(A)$ is assumed to be $>4$, Theorem 2.3 yields $s-\mu(s)=0$, which contradicts the assumption $\operatorname{deg}(A)>2$. Therefore, $\lambda \neq 0$.

Now define $\varphi: S \rightarrow S^{\prime}$ by

$$
\varphi(s)=\lambda \theta(s)+\frac{1}{2} \mu(s), \quad s \in S .
$$


Using (1) one can check that $\varphi\left(s^{2}\right)-\varphi(s)^{2} \in F$ for all $s \in S$. Hence $\varphi(s \circ t)=\varphi(s) \circ \varphi(t)+\tau(s, t)$ for all $s, t \in S$, where $s \circ t=s t+t s$ and $\tau(s, t) \in F$. Since $(s \circ s) \circ(s \circ s)=\{(s \circ s) \circ s\} \circ s$ for all $s \in S$, we have

$$
\begin{aligned}
0= & \varphi([(s \circ s) \circ(s \circ s)-\{(s \circ s) \circ s\} \circ s]) \\
= & \{\varphi(s) \circ \varphi(s)+\tau(s, s)\} \circ\{\varphi(s) \circ \varphi(s)+\tau(s, s)\}+\tau(s \circ s, s \circ s) \\
& -\{\{\varphi(s) \circ \varphi(s)+\tau(s, s)\} \circ \varphi(s)+\tau(s \circ s, s)\} \circ \varphi(s)+\tau(\{s \circ s\} \circ s, s) \\
= & 4 \tau(s, s) \varphi(s)^{2}-4 \tau\left(s^{2}, s\right) \varphi(s)+2 \tau(s, s)^{2}+4 \tau\left(s^{2}, s^{2}\right)-4 \tau\left(s^{3}, s\right)
\end{aligned}
$$

for all $s \in S$. Substituting $\lambda \theta(s)+\frac{1}{2} \mu(s)$ for $\varphi(s)$ we see that

$$
\left[\lambda \tau(s, s) \theta(s)-\left(\tau\left(s^{2}, s\right)-\tau(s, s) \mu(s)\right)\right] \theta(s) \in F
$$

for all $s \in S$. Using Theorem 2.3 twice we conclude that $\tau(s, s)=0$ for all $s \in S$ and so $\varphi$ is a Jordan homomorphism. Let us show that $\varphi$ is bijective. Basically we shall just repeat arguments given at the end of the proof of $\left[10\right.$, Theorem 2]. Suppose that $\varphi(s)=0$. Then $\theta(s)=-\frac{1}{2 \lambda} \mu(s) \in F$. Since $0 \neq \theta(1) \in F_{s}, \theta\left(F_{s}\right)=F_{s}$, and so $s \in F_{s}$. Therefore the linearity of $\varphi$ implies that $\varphi(1)=0$. Since $\varphi$ is a Jordan homomorphism, this yields

$$
2 \varphi(t)=\varphi(1 \circ t)=\varphi(1) \circ \varphi(t)=0 \quad \text { for all } t \in S
$$

forcing $\theta(S) \subseteq F_{s}$ and so $S^{\prime}=F_{s}$, a contradiction. Hence $\varphi$ is injective. Further, $2 \varphi(1)=\varphi(1) \circ \varphi(1)$ and $\varphi(1) \in F_{s}$ together yield that $\varphi(1)=1$. Since $\varphi$ is linear, $\varphi(\sigma)=\sigma$ for all $\sigma \in F_{s}$. It is now straightforward to check that $\theta(s)=\varphi\left(\lambda^{-1} s-\frac{1}{2} \lambda^{-1} \mu(s)\right)$ for all $s \in S$ and so $\varphi$ is a Jordan isomorphism of $S$ onto $S^{\prime}$. Now it follows from [17] (see also [21, 22]) that $\varphi$ can be extended to a surjective $*$-linear homomorphism (which we also denote by $\varphi$ ) of associative $F_{s^{-}}$algebras $\langle S\rangle$ and $\left\langle S^{\prime}\right\rangle$. If $I=\operatorname{ker}(\varphi)$, then $I^{*}=I$ and $I \cap S=0$. Therefore $x^{*}+x \in I \cap S=0$ for all $x \in I$ and so $x^{2} \in I \cap S=0$ for all $x \in I$. On the other hand, the ring $\langle S\rangle$ is prime [18, Theorem 3.4], and so $I=0$. That is, $\varphi$ is an isomorphism.

Let us finally mention that the bound $\operatorname{deg}\left(A^{\prime}\right)>8$ in the theorem is not the best possible. For example, one can lower it to $\operatorname{deg}\left(A^{\prime}\right)>6$ arguing similarly as in the proof [10, Theorem 2]. However, this makes the proof considerably longer.

\section{Normal-preservers: The case of involution of the second kind.}

Theorem 4.1. Let $A$ be $A^{\prime}$ be centrally closed prime algebras over a field $F$ with involution of the second kind. Suppose that $\operatorname{deg}(A)>2, \operatorname{deg}\left(A^{\prime}\right)>2$, and that $\operatorname{char}(F) \neq 2,3$. Let $\theta: A \rightarrow A^{\prime}$ be a bijective $F$-linear map with the property that $\theta(x)$ is normal whenever $x \in A$ is normal. Then $\theta$ is of the form $\theta(x)=\alpha \phi(x)+\beta(x)$ where $\alpha \in F, \alpha \neq 0, \beta: A \rightarrow F$ is a linear map and $\phi$ is either $a$ *-isomorphism or a *-antiisomorphism of $A$ onto $A^{\prime}$. 
Proof. Let $\epsilon \in F$ be such that $\epsilon^{*}=-\epsilon$. Then $A=S+\epsilon S$.

First we show that $\theta(1) \in F$, that is, that $\theta(1)$ is a central element in $A^{\prime}$. Since $s+\lambda$ is a normal element for every $s \in S$ and $\lambda \in F$, it follows that $\theta(s+\lambda)=\theta(s)+\lambda \theta(1)$ is normal, that is, $\left[\theta(s)+\lambda \theta(1), \theta(s)^{*}+\lambda^{*} \theta(1)^{*}\right]=0$ and hence $\lambda\left[\theta(1), \theta(s)^{*}\right]+\lambda^{*}\left[\theta(s), \theta(1)^{*}\right]=0$. First setting $\lambda=1$ and then $\lambda=\epsilon$ it follows that $\left[\theta(1), \theta(s)^{*}\right]=0$, which in turn implies that $\theta(1)$ is central for $A=S+\epsilon S$ and $\theta$ is bijective.

Next, $s^{2}+\lambda s$ is normal for $s \in S$ and $\lambda \in F$, and so $\theta\left(s^{2}\right)+\lambda \theta(s)$ is normal which implies that $\left[\theta\left(s^{2}\right), \theta(s)^{*}\right]=0$. Linearizing we get

$$
\left[\theta\left(s^{2}\right), \theta(t)^{*}\right]+\left[\theta(s \circ t), \theta(s)^{*}\right]=0 \quad \text { for all } s, t \in S .
$$

Again using $A=S+\epsilon S$ it follows easily that $\left[\theta\left(x^{2}\right), \theta\left(x^{*}\right)^{*}\right]=0$ for all $x \in A$. Replacing $x$ by $x+1$ and using the fact that $\theta(1)$ is central it follows that $\left[\theta(x), \theta\left(x^{*}\right)^{*}\right]=0$ for all $x \in A$. Now, using Theorem 2.1 we see that there is $\tau \in F$ and a map $\zeta: A \rightarrow F$ such that $\theta\left(x^{*}\right)^{*}=\tau \theta(x)+\zeta(x), x \in A$. Of course, $\tau \neq 0$ for otherwise $A^{\prime}$ would be commutative, contrary to the assumption. Consequently, $\left[\theta\left(x^{2}\right), \theta(x)\right]=0$ for all $x \in A$. Thus, all the requirements of $[\mathbf{1 0}$, Theorem 2] are fulfilled, and so it follows that $\theta$ is of the form $\theta(x)=\alpha \phi(x)+\beta(x)$ where $\alpha \in F, \alpha \neq 0, \beta$ is a linear map from $A$ into the center of $A^{\prime}$ and $\phi$ is either an isomorphism or an antiisomorphism of $A$ onto $A^{\prime}$.

All it remains to show is that $\phi\left(x^{*}\right)=\phi(x)^{*}, x \in A$.

Assume that $\phi$ is an isomorphism. Then $\psi(x)=\phi\left(x^{*}\right)^{*}$ also defines an isomorphism. We want to show that $\phi=\psi$. We have

$$
\alpha^{*} \psi(x)+\beta\left(x^{*}\right)^{*}=\theta\left(x^{*}\right)^{*}=\tau \theta(x)+\zeta(x)=\tau \alpha \phi(x)+\tau \beta(x)+\zeta(x) .
$$

Since $\alpha \neq 0$ and $\tau \neq 0$ it follows that $\nu(x)=\psi(x)-\gamma \phi(x) \in F$ for every $x \in A$, where $\gamma=\frac{\alpha \tau}{\alpha^{*}}$ is a nonzero element in $F$. Whence

$$
\nu(x y)=\psi(x) \psi(y)-\gamma \phi(x) \phi(y)=\nu(x) \psi(y)+\gamma \phi(x)(\psi(y)-\phi(y)) .
$$

Commuting this expression with $\psi(y)$ it follows, since $\gamma \neq 0$ and $\psi(y)-\phi(y)$ commutes with $\psi(y)$, that $[\phi(x), \psi(y)](\psi(y)-\phi(y))=0$ for all $x, y \in A$. Replacing $x$ by $x z$ we get at once that $\left[A^{\prime}, \psi(y)\right] A^{\prime}(\psi(y)-\phi(y))=0$ for every $y \in A$. Since $A^{\prime}$ is prime this shows that given $y \in A$, either $\psi(y)$ is central or $\psi(y)=\phi(y)$. Since a group cannot be the union of two proper subgroups and since $A^{\prime}$ is noncommutative, it follows that $\psi(y)=\phi(y)$ for all $y \in A$. Similarly we discuss the case when $\phi$ is an antiisomorphism.

\section{Normal-preservers: The case of involution of the first kind.}

Theorem 5.1. Let $A$ be $A^{\prime}$ be centrally closed prime algebras over a field $F$ with involution of the first kind. Suppose that $\operatorname{deg}(A)>6, \operatorname{deg}\left(A^{\prime}\right)>13$ and $\operatorname{char}(F) \neq 2,3$. Further, let $\theta: A \rightarrow A^{\prime}$ be a bijective $*$-linear map with the property that $\theta(x)$ is normal whenever $x \in A$ is normal. Then there exist 
$\mu_{1}, \mu_{2} \in F, \mu_{1} \neq \pm \mu_{2}$, a linear map $\omega:\langle K\rangle \rightarrow F$, and a *-isomorphism $\psi$ of $\langle K\rangle$ onto $\left\langle K^{\prime}\right\rangle$ such that $\theta(x)=\psi\left(\mu_{1} x+\mu_{2} x^{*}\right)+\omega\left(x+x^{*}\right)$ for all $x \in\langle K\rangle$.

First note that, since $\theta$ is $*$-linear, the condition that $\theta$ maps normal elements into normal elements is equivalent to the condition that $\theta(s)$ and $\theta(k)$ commute whenever $s \in S$ and $k \in K$ commute. In particular, for any $k \in K, k^{2}$ is a symmetric element commuting with skew elements $k$ and $k^{3}$, so that $\left[\theta\left(k^{2}\right), \theta(k)\right]=0$ and $\left[\theta\left(k^{2}\right), \theta\left(k^{3}\right)\right]=0$. One can note from the proof that this is essentially all that we need; more precisely, in the theorem we could replace the condition that $\theta$ preserves normal elements by a milder condition that $\theta$ satisfies these two identities and that $\theta(F)=F$.

The proof of Theorem 5.1 is broken up into a series of lemmas. We begin with:

Lemma 5.2. There exists $\lambda_{0} \neq 0$ in $F$ such that the map $\phi=\lambda_{0} \theta$ satisfies $\phi\left(k^{2}\right)-\phi(k)^{2} \in F$ for all $k \in K$.

Proof. As already observed, $\left[\theta(k), \theta\left(k^{2}\right)\right]=0$ for all $k \in K$. But then it follows from Theorem 2.2 that there exist $\lambda_{0} \in F$ and a linear map $\mu_{0}: K \rightarrow F$ such that $\theta\left(k^{2}\right)-\lambda_{0} \theta(k)^{2}-\mu_{0}(k) \theta(k) \in F$ for every $k \in K$. However, since $\theta$ is $*$-linear and $*$ is of the first kind, $\mu_{0}$ must be zero. Hence we see that $\phi=\lambda_{0} \theta$ indeed satisfies $\phi\left(k^{2}\right)-\phi(k)^{2} \in F, k \in K$. Finally, assuming that $\lambda_{0}$ is zero we arrive at $\theta\left(k^{2}\right) \in F$; however, $\theta(F)=F$ for $\theta(1) \in F$ (namely, $\theta(1)$ commutes with $K^{\prime}=\theta(K)$ and $\operatorname{deg}\left(A^{\prime}\right)>2$ - cf. the proof of Theorem 2.2), and so $k^{2}$ lies in $F$ for every $k \in K$. But Theorem 2.3 tells us that this is impossible. The lemma is thereby proved.

Of course, $\phi$ has the same properties as $\theta$, that is, it is $*$-linear, bijective and preserves normal elements.

Define $\epsilon: K \times K \rightarrow F$ by

$$
\epsilon(k, l)=\frac{1}{2}\{\phi(k \circ l)-\phi(k) \circ \phi(l)\} .
$$

Clearly, $\epsilon$ is a bilinear symmetric map.

Lemma 5.3. There exist $\lambda \neq 0$ in $F$ and a symmetric bilinear map $\mu$ : $K \times K \rightarrow F$ such that

$$
\phi(k l k)=\lambda \phi(k) \phi(l) \phi(k)+\mu(k, l) \phi(k)
$$

for all $k, l \in K$.

Proof. If $k \in K$, then $k^{2} \in S$ and $k^{3} \in K$, so that $\left[\phi\left(k^{3}\right), \phi\left(k^{2}\right)\right]=0$. However, $\phi\left(k^{2}\right)=\phi(k)^{2}+\epsilon(k, k)$ and so $\left[\phi\left(k^{3}\right), \phi(k)^{2}\right]=0$ for every $k \in K$. Since $\operatorname{deg}\left(A^{\prime}\right)>10$, Theorem 2.2, together with the fact that $\phi$ is $*$-linear and $*$ is of the first kind, shows that there are $\lambda \in F$ and a symmetric bilinear map $\mu: K \times K \rightarrow F$ such that

$$
\phi\left(k^{3}\right)=\lambda \phi(k)^{3}+\mu(k, k) \phi(k) \quad \text { for all } k \in K .
$$


Note that $\lambda=0$ yields $k^{3}=\mu(k, k) k$ which is, since $\operatorname{deg}(A)>6$, impossible by Theorem 2.3. Thus, $\lambda \neq 0$.

Linearizing (3) we get

$$
\begin{aligned}
\phi\left(k^{2} l+k l k+l k^{2}\right)= & \lambda\left\{\phi(k)^{2} \phi(l)+\phi(k) \phi(l) \phi(k)+\phi(l) \phi(k)^{2}\right\} \\
& +\mu(k, k) \phi(l)+2 \mu(k, l) \phi(k) .
\end{aligned}
$$

Next we compute $\phi\left(k^{2} l k+k l k^{2}\right)$ in two different ways. First using (2) we get

$$
\begin{aligned}
& 2 \phi\left(k^{2} l k+k l k^{2}\right) \\
& =\phi\left(k \circ\left\{k^{2} \circ l+k l k\right\}\right)-\phi\left(k^{3} \circ l\right) \\
& =\phi(k) \circ \phi\left(k^{2} \circ l+k l k\right)+2 \epsilon\left(k, k^{2} \circ l+k l k\right)-\phi\left(k^{3}\right) \circ \phi(l)-2 \epsilon\left(k^{3}, l\right),
\end{aligned}
$$

so that

$$
\begin{aligned}
2 \phi\left(k^{2} l k+k l k^{2}\right)- & \phi(k) \phi\left(k^{2} l+k l k+l k^{2}\right) \\
& -\phi\left(k^{2} l+k l k+l k^{2}\right) \phi(k)+\phi\left(k^{3}\right) \phi(l)+\phi(l) \phi\left(k^{3}\right) \in F .
\end{aligned}
$$

Applying (3) and (4) it follows that

$$
\begin{aligned}
& 2 \phi\left(k^{2} l k+k l k^{2}\right) \\
& -\phi(k)\left\{\lambda \phi(k)^{2} \phi(l)+\lambda \phi(k) \phi(l) \phi(k)+\lambda \phi(l) \phi(k)^{2}\right. \\
& \quad+\mu(k, k) \phi(l)+2 \mu(k, l) \phi(k)\} \\
& -\left\{\lambda \phi(k)^{2} \phi(l)+\lambda \phi(k) \phi(l) \phi(k)+\lambda \phi(l) \phi(k)^{2}\right. \\
& \quad+\mu(k, k) \phi(l)+2 \mu(k, l) \phi(k)\} \phi(k) \\
& +\left\{\lambda \phi(k)^{3}+\mu(k, k) \phi(k)\right\} \phi(l)+\phi(l)\left\{\lambda \phi(k)^{3}+\mu(k, k) \phi(k)\right\} \in F,
\end{aligned}
$$

and hence

$$
\phi\left(k^{2} l k+k l k^{2}\right)-\lambda\left(\phi(k)^{2} \phi(l) \phi(k)+\phi(k) \phi(l) \phi(k)^{2}\right)-2 \mu(k, l) \phi(k)^{2} \in F .
$$

On the other hand, (2) implies that

$$
\phi\left(k^{2} l k+k l k^{2}\right)=\phi(k \circ\{k l k\})=\phi(k) \circ \phi(k l k)+2 \epsilon(k, k l k) .
$$

Comparing we obtain

$$
\phi(k) \circ\{\phi(k l k)-\lambda \phi(k) \phi(l) \phi(k)-\mu(k, l) \phi(k)\} \in F
$$

for all $k, l \in K$. According to the statement (ii) of Theorem 2.2 we must then have $\phi(k l k)-\lambda \phi(k) \phi(l) \phi(k)-\mu(k, l) \phi(k)=0$ for all $k, l \in K$, and the lemma is proved. 
We shall need the conclusion of Lemma 5.3 in the following form

$$
\begin{aligned}
\phi\left(k_{1} l k_{2}+k_{2} l k_{1}\right)= & \lambda\left\{\phi\left(k_{1}\right) \phi(l) \phi\left(k_{2}\right)+\phi\left(k_{2}\right) \phi(l) \phi\left(k_{1}\right)\right\} \\
& +\mu\left(k_{1}, l\right) \phi\left(k_{2}\right)+\mu\left(k_{2}, l\right) \phi\left(k_{1}\right)
\end{aligned}
$$

for all $k_{1}, k_{2}, l \in K$.

Lemma 5.4. $\mu(k, l)=0$ for all $k, l \in K$.

Proof. The proof is based on computing $\phi\left(k l_{1} k l_{2} k+k l_{2} k l_{1} k\right)$, where $k, l_{1}, l_{2}$ are arbitrary elements in $K$, in two different ways. First, applying (5) we get

$$
\begin{aligned}
\phi & \left(k l_{1} k l_{2} k+k l_{2} k l_{1} k\right) \\
= & \phi\left(\left(k l_{1} k\right) l_{2} k+k l_{2}\left(k l_{1} k\right)\right) \\
= & \lambda\left\{\phi\left(k l_{1} k\right) \phi\left(l_{2}\right) \phi(k)+\phi(k) \phi\left(l_{2}\right) \phi\left(k l_{1} k\right)\right\}+\mu\left(k l_{1} k, l_{2}\right) \phi(k) \\
& +\mu\left(k, l_{2}\right) \phi\left(k l_{1} k\right) \\
= & \lambda^{2}\left\{\phi(k) \phi\left(l_{1}\right) \phi(k) \phi\left(l_{2}\right) \phi(k)+\phi(k) \phi\left(l_{2}\right) \phi(k) \phi\left(l_{1}\right) \phi(k)\right\} \\
& +2 \lambda \mu\left(k, l_{1}\right) \phi(k) \phi\left(l_{2}\right) \phi(k)+\lambda \mu\left(k, l_{2}\right) \phi(k) \phi\left(l_{1}\right) \phi(k) \\
& +\left\{\mu\left(k l_{1} k, l_{2}\right)+\mu\left(k, l_{2}\right) \mu\left(k, l_{1}\right)\right\} \phi(k) .
\end{aligned}
$$

However, $l_{1}$ and $l_{2}$ appear symmetrically in the expression $k l_{1} k l_{2} k+k l_{2} k l_{1} k$ and so, on the other hand, we must have

$$
\begin{aligned}
\phi & \left(k l_{1} k l_{2} k+k l_{2} k l_{1} k\right) \\
= & \lambda^{2}\left\{\phi(k) \phi\left(l_{2}\right) \phi(k) \phi\left(l_{1}\right) \phi(k)+\phi(k) \phi\left(l_{1}\right) \phi(k) \phi\left(l_{2}\right) \phi(k)\right\} \\
& +2 \lambda \mu\left(k, l_{2}\right) \phi(k) \phi\left(l_{1}\right) \phi(k)+\lambda \mu\left(k, l_{1}\right) \phi(k) \phi\left(l_{2}\right) \phi(k) \\
& +\left\{\mu\left(k l_{2} k, l_{1}\right)+\mu\left(k, l_{1}\right) \mu\left(k, l_{2}\right)\right\} \phi(k) .
\end{aligned}
$$

Comparing both relations we obtain

$$
\lambda \phi(k)\left\{\mu\left(k, l_{2}\right) \phi\left(l_{1}\right)-\mu\left(k, l_{1}\right) \phi\left(l_{2}\right)\right\} \phi(k)=\left\{\mu\left(k l_{1} k, l_{2}\right)-\mu\left(k l_{2} k, l_{1}\right)\right\} \phi(k)
$$

for all $k, l_{1}, l_{2} \in K$. Now using Theorem 2.3 twice it follows that

$$
\mu\left(k, l_{2}\right) \phi\left(l_{1}\right)-\mu\left(k, l_{1}\right) \phi\left(l_{2}\right)=0 \quad \text { for all } k, l_{1}, l_{2} \in K,
$$

which readily implies the assertion of the lemma.

Thus, (5) now reduces to

$$
\phi\left(k_{1} l k_{2}+l_{2} k l_{1}\right)=\lambda\left\{\phi\left(k_{1}\right) \phi(l) \phi\left(k_{2}\right)+\phi\left(k_{2}\right) \phi(l) \phi\left(k_{1}\right)\right\} .
$$


Lemma 5.5. There exists $\rho \in F$ such that $\rho^{2}=\lambda$ and $\phi([k, l])=$ $\rho[\phi(k), \phi(l)]$ for all $k, l \in K$.

Proof. We have arrived at the situation when Theorem 2.4 can be applied. Taking a polynomial $f$ to be $f\left(x_{1}, x_{2}, x_{3}\right)=x_{1} x_{2} x_{3}+x_{3} x_{2} x_{1}$ and a map $B$ to be equal to $B(k, l)=\phi([k, l])$, we see, by making use of $(6)$, that all the conditions of this theorem are fulfilled (this is the place when the condition $\operatorname{deg}\left(A^{\prime}\right)>13$ is used). It follows that there exists $\rho \in F$ such that $\phi([k, l])-\rho[\phi(k), \phi(l)] \in F$ for all $k, l \in K$; however, since $*$ is of the first kind, this clearly yields $\phi([k, l])=\rho[\phi(k), \phi(l)]$. It remains to show that $\rho^{2}=\lambda$. We have

$$
\phi\left(\left[\left[k_{1}, k_{2}\right], k_{3}\right]\right)=\rho\left[\phi\left(\left[k_{1}, k_{2}\right]\right), \phi\left(k_{3}\right)\right]=\rho^{2}\left[\left[\phi\left(k_{1}\right), \phi\left(k_{2}\right)\right], \phi\left(k_{3}\right)\right] .
$$

On the other hand, using (6), we get

$$
\begin{aligned}
\phi\left(\left[\left[k_{1}, k_{2}\right], k_{3}\right]\right) & =\phi\left(k_{1} k_{2} k_{3}+k_{3} k_{2} k_{1}\right)-\phi\left(k_{2} k_{1} k_{3}+k_{3} k_{1} k_{2}\right) \\
& =\lambda\left[\left[\phi\left(k_{1}\right), \phi\left(k_{2}\right)\right], \phi\left(k_{3}\right)\right] .
\end{aligned}
$$

Whence $\left(\rho^{2}-\lambda\right)\left[\left[K^{\prime}, K^{\prime}\right], K^{\prime}\right]=0$. Suppose that $\left[\left[K^{\prime}, K^{\prime}\right], K^{\prime}\right]=0$. Then applying [8, Theorem 9.1.13] we get that $\operatorname{deg}\left(A^{\prime}\right) \leq 2$, a contradiction. Therefore $\rho^{2}=\lambda$ and the lemma is proved.

Lemma 5.6. There exist a *-isomorphism $\psi$ of an algebra $\langle K\rangle$ onto an algebra $\left\langle K^{\prime}\right\rangle$ and a linear map $\tau: K \circ K \rightarrow F$ such that $\psi(k)=\rho \phi(k)$ for all $k \in K$ and $\psi(s)=\lambda \phi(s)-\tau(s)$ for all $s \in K \circ K$.

Proof. We first define $\psi$ on $K$ by $\psi(k)=\rho \phi(k), k \in K$. Since $\phi=\lambda_{0} \theta$ and $\theta$ is a $*$-linear map, $\psi(K)=\theta(K)=K^{\prime}$. It follows from Lemmas 5.3, 5.4 and 5.5 together that

$$
\psi([k, l])=[\psi(k), \psi(l)] \quad \text { and } \quad \psi\left(k^{3}\right)=\psi(k)^{3} \quad \text { for all } k, l \in K \text {. }
$$

Now both (7) and [8, Lemma 9.4.5] imply that $\psi$ can be uniquely extended to an isomorphism (which we also denote by $\psi$ ) of associative rings $\langle K\rangle$ and $\left\langle K^{\prime}\right\rangle$. Since $\left.\psi\right|_{K}$ is a linear map and $K$ generates $\langle K\rangle, \psi$ is an isomorphism of algebras. Clearly

$$
\psi(K)=K^{\prime} \quad \text { and } \quad \psi(K \circ K)=\psi(K) \circ \psi(K)=K^{\prime} \circ K^{\prime} .
$$


According to [8, Lemma 9.1.5], $\langle K\rangle=K+K \circ K$ and $\left\langle K^{\prime}\right\rangle=K^{\prime}+K^{\prime} \circ$ $K^{\prime}$. Obviously $K$ (respectively, $K \circ K$ ) is the set of skew (respectively, symmetric) elements of the algebra $\langle K\rangle$. It now follows from (8) that $\psi$ is a $*$-isomorphism.

Now define a linear map $\tau$ on $K \circ K$ by $\tau(s)=\lambda \phi(s)-\psi(s)$. We claim that $\tau(s)$ lies in $F$ for any $s \in K \circ K$. Indeed, clearly the vector space $K \circ K$ is spanned by the set $\left\{k^{2} \mid k \in K\right\}$. Given $k \in K$, we have

$$
\begin{aligned}
\tau\left(k^{2}\right) & =\lambda \phi\left(k^{2}\right)-\psi\left(k^{2}\right)=\lambda \phi\left(k^{2}\right)-\psi(k)^{2} \\
& =\lambda \phi\left(k^{2}\right)-\{\rho \phi(k)\}^{2}=\lambda\left\{\phi\left(k^{2}\right)-\phi(k)^{2}\right\} \in F
\end{aligned}
$$

by Lemma 5.2 which proves our claim.

Finally, invoking the definition of $\phi$ we see from Lemma 5.6 that for any $x \in\langle K\rangle$ we have

$$
\begin{aligned}
\theta(x) & =\lambda_{0} \phi(x)=\frac{\lambda_{0}}{2} \phi\left(x-x^{*}\right)+\frac{\lambda_{0}}{2} \phi\left(x+x^{*}\right) \\
& =\frac{\lambda_{0} \rho^{-1}}{2} \psi\left(x-x^{*}\right)+\frac{\lambda_{0} \lambda^{-1}}{2} \psi\left(x+x^{*}\right)+\frac{\lambda_{0} \lambda^{-1}}{2} \tau\left(x+x^{*}\right) .
\end{aligned}
$$

Now set $\mu_{1}=\frac{1}{2} \lambda_{0}\left(\lambda^{-1}+\rho^{-1}\right), \mu_{2}=\frac{1}{2} \lambda_{0}\left(\lambda^{-1}-\rho^{-1}\right), \omega\left(x-x^{*}\right)=0, \omega(x+$ $\left.x^{*}\right)=\frac{1}{2} \lambda_{0} \lambda^{-1} \tau\left(x+x^{*}\right)$ and note that the desired conclusion holds true.

Acknowledgement. The paper has been written while the second and the third author were visiting the National Cheng-Kung University. They would like to express their deep gratitude to the University for its hospitality and for the financial support of their visit.

The authors would also like to thank the referee for careful reading of the paper and for some useful suggestions.

\section{References}

[1] A survey of linear preserver problems, Linear and Multilinear Algebra, 33(1-2) (1992), Gordon and Breach, Yverdon, 1992, 1-129, MR 96c:15043.

[2] R. Banning and M. Mathieu, Commutativity preserving mappings on semiprime rings, Comm. Algebra, 25 (1997), 247-265, MR 97j:16033, Zbl 0865.16015.

[3] K.I. Beidar, On functional identities and commuting additive mappings, Comm. Algebra, 26 (1998), 1819-1850, MR 99f:16023, Zbl 0901.16011.

[4] K.I. Beidar, S.-C. Chang, M.A. Chebotar and Y. Fong, On functional identities in left ideals of prime rings, Comm. Algebra, 28 (2000), 3041-3058, MR 2001c:16045, Zbl 0971.16014.

[5] K.I. Beidar and M.A. Chebotar, On functional identities and d-free subsets of rings I, Comm. Algebra, 28 (2000), 3925-3952, MR 2001j:16046. 
[6] _ On functional identities and d-free subsets of rings II, Comm. Algebra, 28 (2000), 3953-3972, MR 2001j:16046.

[7] K.I. Beidar and W.S. Martindale 3rd, On functional identities in prime rings with involution, J. Algebra, 203 (1998), 491-532, MR 99f:16024, Zbl 0904.16012.

[8] K.I. Beidar, W.S. Martindale 3rd and A.V. Mikhalev, Rings with Generalized Identities, Marcel Dekker, Inc., New York-Basel-Hong Kong, 1996, MR 97g:16035, Zbl 0847.16001.

[9] M. Brešar, Centralizing mappings and derivations in prime rings, J. Algebra, 156 (1993), 385-394, MR 94f:16042, Zbl 0773.16017.

[10] _ Commuting traces of biadditive mappings, commutativity-preserving mappings and Lie mappings, Trans. Amer. Math. Soc., 335 (1993), 525-546, MR 93d:16044, Zbl 0791.16028.

[11] _ Functional identities: A survey, Contemporary Math., 259 (2000), 93-109, MR 2001h:16023, Zbl 0967.16011.

[12] M. Brešar and P. Šemrl, Normal-preserving linear mappings, Can. Math. Bull., 37 (1994), 306-309, MR 96b:47040, Zbl 0816.47018.

[13] _ Linear preservers on $\mathcal{B}(X)$, Banach Center Publ., 38 (1997), 49-58, MR 99c:47044, Zbl 0939.47031.

[14] G.H. Chan and M.H. Lim, Linear transformations on symmetric matrices that preserve commutativity, Linear Algebra Appl., 47 (1982), 11-22, MR 83k:15020, Zbl 0492.15006.

[15] M.D. Choi, A.A. Jafarian and H. Radjavi, Linear maps preserving commutativity, Linear Algebra Appl., 87 (1987), 227-242, MR 88f:15003, Zbl 0615.15004.

[16] C.M. Kunicki and R.D. Hill, Normal-preserving linear transformations, Linear Algebra Appl., 170 (1992), 107-115, MR 93j:15004, Zbl 0751.15002.

[17] L.A. Lagutina, Jordan homomorphisms of associative algebras with involution, Algebra and Logic, 27 (1988), 250-260, MR 90k:16013, Zbl 0697.16013.

[18] C. Lanski, On the relationship of a ring and the subring generated by its symmetric elements, Pacific J. Math., 44 (1973), 581-592, MR 48 \#331, Zbl 0252.16004.

[19] C.-K. Li and N.-K. Tsing, Linear preserver problems: A brief introduction and some special techniques, Linear Algebra Appl., 162-164 (1992), 217-235, MR 93b:15003, Zbl 0762.15016.

[20] M. Marcus, Linear operations on matrices, Amer. Math. Monthly, 69 (1962), 837-847, MR 26 \#5007, Zbl 0108.01104.

[21] W.S. Martindale 3rd, Jordan homomorphisms onto nondegenerate Jordan algebras, J. Algebra, 133 (1990), 500-511, MR 91m:17045, Zbl 0708.17027.

[22] K. McCrimmon, The Zelmanov approach to Jordan homomorphisms of associative algebras, J. Algebra, 123 (1989), 457-477, MR 90j:17053, Zbl 0675.17016.

[23] C. Procesi, Rings with Polynomial Identities, Marcel Decker, Inc., 1973, MR 51 \#3214, Zbl 0262.16018.

[24] H. Radjavi, Commutativity-preserving operators on symmetric matrices, Linear Algebra Appl., 61 (1984), 219-224, MR 85h:15007, Zbl 0547.15007.

[25] H. Radjavi and P. Rosenthal, Invariant Subspaces, Ergeb. Math. Grenzeb., 77, Springer-Verlag, New York, 1973, MR 51 \#3924, Zbl 0269.47003. 
[26] L.H. Rowen, Polynomial Identities in Ring Theory, Academic Press, 1980, MR 82a:16021, Zbl 0461.16001.

Received September 25, 2000 and revised January 10, 2001. The second author was partially supported by a grant from the Ministry of Science of Slovenia.

Department of Mathematics

National Cheng-Kung University

TAINAN

TAIWAN

E-mail address: beidar@mail.ncku.edu.tw

Department of Mathematics, PF

UNIVERSITY OF MARIBOR

MARIBOR

SLOVENIA

E-mail address: bresar@uni-mb.si

Department of Mechanics and Mathematics

Tula State University

TUlA

Russia

E-mail address: mchebotar@tula.net

Department of Mathematics

National Cheng-Kung University

TAINAN

TAIWAN

E-mail address: fong@mail.ncku.edu.tw 